\title{
A 24-Week, Randomized, Controlled Study to Evaluate the Tolerability, Safety and Efficacy of 2 Different Titration Schemes of the Rivastigmine Patch in Japanese Patients with Mild to Moderate Alzheimer's Disease
}

\author{
Yu Nakamura ${ }^{b}$ Christine Strohmaier ${ }^{a}$ Kaoru Tamura ${ }^{c} \quad$ Naoko Kataoka $^{c}$ \\ Masayuki Nakano ${ }^{e}$ Shoichiro Oda ${ }^{e}$ Kazuma Nishimura e Akira Hommad \\ ${ }^{a}$ Novartis Pharma AG, Basel, Switzerland; ${ }^{b}$ Department of Neuropsychiatry, Faculty of \\ Medicine, Kagawa University, Kagawa, ' Novartis Pharma K.K., and d Dementia Care Research \\ and Training Center, Tokyo, and eOno Pharmaceutical Co., Ltd., Osaka, Japan
}

\section{Key Words}

Alzheimer's disease - Cholinesterase inhibitors - Japan · Randomized clinical trial ·

Rivastigmine patch · Titration scheme

\section{Abstract}

Aim: To investigate whether 1-step titration of the rivastigmine patch (initiated at $5 \mathrm{~cm}^{2}$ and titrated to $10 \mathrm{~cm}^{2}$ after 4 weeks) is well tolerated in Japanese patients with Alzheimer's disease (AD) as compared to 3-step titration (initiated at $2.5 \mathrm{~cm}^{2}$ and titrated by $2.5 \mathrm{~cm}^{2}$ every 4 weeks to $10 \mathrm{~cm}^{2}$ ). Methods: A 24-week, multicenter, randomized, double-blind study was conducted in Japan between July 2012 and May 2014. Patients with mild to moderate AD aged 50-85 years were randomized 1:1 to 1-step or 3-step titration of the rivastigmine once-daily patch. The primary endpoint was the proportion of patients with adverse events leading to discontinuation. Results: Of 216 patients randomized, 215 (1-step, $n=107 ; 3$-step, $n=108$ ) were included in the safety analysis. The primary endpoint outcome was $15.0 \%$ in the 1 -step group and $18.5 \%$ in the 3 -step group. The observed treatment difference was $-3.6 \%$ (95\% confidence interval: -17.0, 9.6), falling within the prespecified acceptance range. Conclusion: The tolerability of two different titration schemes was similar in Japanese patients with AD. 


\section{Introduction}

Alzheimer's disease (AD) is the most prevalent cause of dementia and a progressive neurodegenerative disorder characterized by cognitive dysfunction. The estimated number of dementia patients aged $\geq 65$ in Japan was over 4.6 million in 2013 according to Grants-inAid for Scientific Research from the Ministry of Health, Labour and Welfare. In Japan, three acetylcholine esterase (AChE) inhibitors (rivastigmine, donepezil and galantamine) and an $\mathrm{N}$-methyl-D-aspartate receptor antagonist (memantine) have been approved for AD treatment.

Rivastigmine is a dual inhibitor of AChE and butyrylcholinesterase and shows preferential selectivity for cholinesterase isoforms found in the brain, particularly in neurodegenerating tissue (i.e., the cerebral cortex and the hippocampus). The rivastigmine patch is the first transdermal treatment approved in many countries for AD [1] and, as of 2014, has been used for the treatment of AD in more than 90 countries worldwide.

For all the available drugs for $\mathrm{AD}$ treatments, a titration period, ranging from 1 to 4 weeks in Japan, except for the rivastigmine patch, is required to reach the effective dose. According to the current Japanese label, treatment with the rivastigmine patch starts at $2.5 \mathrm{~cm}^{2}(4.5 \mathrm{mg} /$ day), and its dose should be increased by $4.5 \mathrm{mg} /$ day at 4-week intervals up to the effective dose of $10 \mathrm{~cm}^{2}$ (18 mg/day) based on the design of a pivotal study [2]. Therefore, the currently approved titration scheme of the rivastigmine patch in Japan needs at least 3 steps (a minimum of 12 weeks) before reaching the effective dose, which leads to major concerns for physicians and/or caregivers that dementia symptoms may not be sufficiently treated during the titration period. On the other hand, the alternative schedule of 4-week titration (1-step) of the rivastigmine patch starting with $9 \mathrm{mg} /$ day up to $18 \mathrm{mg} /$ day is already available in most countries worldwide. This shorter and simpler titration could provide patients with faster access to the effective dose and have an earlier effect on their dementia symptoms. However, clinical studies in Japanese AD patients to assess the tolerability, safety and efficacy of the 1-step titration have never been confirmed.

The purpose of this study was to investigate whether the 1-step titration scheme is tolerated, safe and effective in Japanese patients with AD in comparison with the 3-step titration scheme.

\section{Materials and Methods}

\section{Trial Design}

This was a 24-week, multicenter, randomized, double-blind, 2-treatment-arm, parallelgroup study conducted from July 2012 through May 2014 at 49 clinics or hospitals in Japan. The study consisted of a 4-week screening period, a 16-week titration period (weeks 1-16), and an 8-week maintenance period (weeks 17-24) (ClinicalTrials.gov Identifier: NCT01614886; table 1).

\section{Participants}

Male and female (not of child-bearing potential) outpatients aged 50-85 years who were diagnosed with dementia of the Alzheimer's type (according to the Diagnostic and Statistical Manual of Mental Disorders, ed 4) and probable AD (according to the National Institute of Neurological and Communicative Disorders and Stroke and the Alzheimer's Disease and Related Disorders Association) [3] were recruited. Inclusion criteria were mild to moderate $\mathrm{AD}$ on the basis of a score of $\geq 10$ and $\leq 20$ on the Mini-Mental State Examination (MMSE; score range, $0-30$, with higher scores indicating better cognitive function) [4] at randomization and 
Table 1. Study design and interventions

\begin{tabular}{|c|c|c|c|c|c|c|c|c|}
\hline \multirow{5}{*}{$\begin{array}{l}\text { Week } \\
\text { 1-step titration } \\
\text { 3-step titration }\end{array}$} & \multicolumn{2}{|c|}{ Pre-randomization period } & \multicolumn{6}{|c|}{ Double-blind treatment period } \\
\hline & \multirow{2}{*}{$\begin{array}{l}\text { screening } \\
-4 \text { to }-1\end{array}$} & \multirow{2}{*}{$\frac{\text { baseline }^{\mathrm{a}}}{0}$} & \multicolumn{4}{|c|}{ titration } & \multicolumn{2}{|c|}{ maintenance } \\
\hline & & & $1-4$ & $5-8$ & $9-12$ & $13-16$ & $17-20$ & $21-24$ \\
\hline & None & & $9 \mathrm{mg}$ & $18 \mathrm{mg}$ & $18 \mathrm{mg}$ & $18 \mathrm{mg}$ & $18 \mathrm{mg}$ & $18 \mathrm{mg}$ \\
\hline & None & & $4.5 \mathrm{mg}$ & $9 \mathrm{mg}$ & $13.5 \mathrm{mg}$ & $18 \mathrm{mg}$ & $18 \mathrm{mg}$ & $18 \mathrm{mg}$ \\
\hline
\end{tabular}

Patch size: $4.5 \mathrm{mg}=2.5 \mathrm{~cm}^{2}, 9 \mathrm{mg}=5 \mathrm{~cm}^{2}, 13.5 \mathrm{mg}=7.5 \mathrm{~cm}^{2}, 18 \mathrm{mg}=10 \mathrm{~cm}^{2}$.

a The study drug was started on the day after the baseline visit.

sufficient education to read, write and communicate effectively at the premorbid state. Participants had to be willing to cooperate and complete all aspects of the study and be capable of doing so, either alone or with the aid of a responsible caregiver who was required to accept the responsibility for supervising the treatment, assessing the condition of the patient throughout the study, and for providing input to efficacy assessments according to protocol requirements. The responsible caregiver had to directly assess the condition of the patient twice a day (day/night) for 4 days or more per week. Patients had to be resident with someone in the community throughout the study.

Exclusion criteria at the time of randomization included any medical or neurological conditions other than $\mathrm{AD}$ that could possibly result in impaired cognitive function (e.g. syphilis, head injury, Huntington's disease, Parkinson's disease, subdural hematoma, normal pressure hydrocephalus or brain tumor) and an advanced, progressive or unstable disease that could interfere with study assessments or put the patient at special risk (e.g. vascular dementia, major depression, severe cerebrovascular disease or severe cardiovascular disease). The use of rivastigmine in the past or the use of donepezil or galantamine during the 4 weeks prior to randomization was also prohibited. Other exclusion criteria were the initiation, dosage change and administration of memantine during the 4 weeks prior to randomization.

Written informed consent was obtained from each patient and legal representative. If the patient was incapable of signing the informed consent document, a legal representative signed the form, with the assent of the patient. The clinical study was designed, implemented and reported in accordance with good clinical practice, with applicable local regulations and with the ethical principles laid down in the Declaration of Helsinki.

\section{Interventions}

Four different rivastigmine patch strengths and matching placebo patches were used in this study: $4.5 \mathrm{mg}$ (size, $\left.2.5 \mathrm{~cm}^{2}\right), 9 \mathrm{mg}\left(5 \mathrm{~cm}^{2}\right), 13.5 \mathrm{mg}\left(7.5 \mathrm{~cm}^{2}\right)$ and $18 \mathrm{mg}\left(10 \mathrm{~cm}^{2}\right)$. Two patches (1 rivastigmine patch and 1 placebo patch) were applied once daily during weeks 1-12 and 1 rivastigmine 18-mg patch was applied once daily during weeks 13-24 according to the titration schedule. The patches were applied by the caregiver to clean and dry skin on the patient's back (alternatively, the patch could be applied to the upper arm or chest) and had to be worn for $24 \mathrm{~h}$. A diary kept by the caregiver for each patient was used to assess treatment compliance.

After screening, eligible patients were randomly assigned at a ratio of 1:1 to a 1-step or 3 -step titration scheme of the rivastigmine patch. Patients assigned to the 1-step titration scheme were started on $9 \mathrm{mg} /$ day and after 4 weeks uptitrated to the target treatment dose of $18 \mathrm{mg} /$ day. Those assigned to the 3-step titration scheme were started on $4.5 \mathrm{mg} /$ day and 
uptitrated in steps of $4.5 \mathrm{mg} /$ day every 4 weeks to $18 \mathrm{mg} /$ day (table 1). If the target dose was not reached during the titration period, the investigator could increase the dose up to $18 \mathrm{mg} /$ day during the maintenance period. Dose adjustments and interruptions were permitted for patients unable to tolerate the protocol-specified dose until tolerability improved. The patients were maintained at their highest and well-tolerated dose until the end of the study (week 24).

The concomitant use of the following medications was prohibited: any study drug other than the rivastigmine patch; cholinesterase inhibitors (e.g. donepezil, galantamine or marketed rivastigmine patch); selegiline; centrally acting anticholinergic drugs; olanzapine; tricyclic and tetracyclic antidepressants; succinylcholine-type muscle relaxants, and lithium.

In this study, the study drug was discontinued for a given patient if the investigator determined that continuation of the treatment would result in a significant safety risk for that patient based on safety assessments, including adverse events (AEs) and abnormal laboratory values, which were done at each visit throughout the study.

\section{Objectives and Endpoints}

The primary objective of this study was to investigate the tolerability of the 1-step titration scheme versus the 3-step titration scheme by comparing the primary endpoint. The primary endpoint was the proportion of patients with AEs leading to discontinuation during the 24-week study period.

Key secondary objectives were to evaluate the efficacy, safety and treatment retention rate. Efficacy evaluations, which were defined as secondary endpoints, included the 11-item cognitive scale of the Japanese version of the AD Assessment Scale-cognitive subscale (ADAS-J cog, score range, 0-70, with higher scores indicating greater cognitive impairment) [5], MMSE, and the 7-grade investigators impression scale ('markedly improved', 'improved', 'slightly improved', 'no change', 'slightly aggravated', 'aggravated' and 'markedly aggravated') of the Japanese Clinical Global Impression of Change (J-CGIC) [6]. The efficacy assessments were done at baseline and at the end of the study (week 24 or discontinuation). ADAS-J cog was additionally performed at weeks 8 and 16, and J-CGIC was performed at all visits (weeks $4,8,12,16$ and 20). The same rater performed efficacy assessments for a given patient whenever possible. ADAS-J cog was assessed by the raters who completed training prepared by the sponsor and were different from those who evaluated safety.

Safety assessments consisted of collecting all AEs, serious AEs (SAEs), with their severity and relationship to the study drug. They included the regular monitoring of hematology, blood chemistry and urine as well as regular assessments of electrocardiogram (ECG), vital signs, physical condition and weight.

The treatment retention rate was defined as the proportion of patients who met all the following criteria: (1) completed the study, (2) were treated only at $18 \mathrm{mg} /$ day throughout the last 8 weeks of the study, and (3) received $18 \mathrm{mg}$ for $75 \%$ or more of the days during the last 8 weeks of the study.

\section{Sample Size}

The sample size was determined to evaluate the primary objective. Assuming that the proportion of patients with AEs leading to discontinuation (primary endpoint) in both 1-step and 3-step titration schemes is $13 \%$, which was the value observed in the 18-mg treatment group (3-step titration scheme) in the previous phase III study for rivastigmine patch in Japanese patients with mild to moderate AD [2], 100 patients per group in this study would ensure a $>90 \%$ probability of observing the treatment difference falling between the prespecified acceptance range $(-9.0,9.0 \%)$. The value of $9.0 \%$ was chosen based on the $\mathrm{AE}$ 
Nakamura et al.: Different Titration Schemes of the Rivastigmine Patch in Japanese

Patients with Mild to Moderate Alzheimer's Disease

discontinuation rates in previous clinical studies of rivastigmine conducted in and outside Japan $[2,7,8]$.

\section{Randomization}

All eligible patients were randomly assigned to one of the two treatment groups. The randomization numbers were generated by a Contract Research Organization (CRO) that automated the assignment of treatment arms to randomization numbers in the specified ratio using dynamic randomization with weight $(<45,45$ to $<55, \geq 55 \mathrm{~kg})$, MMSE score $(10-15$, 16-20), and concomitant use of memantine (yes, no) as stratification factors. Randomization of the patients was performed using a centralized allocation method. After the investigator at each site had obtained the patient's consent, he or she contacted a CRO staff member who was independent of the recruitment process for allocation consignment via facsimile.

\section{Blinding}

Patients, caregivers, investigator staff, persons performing the assessments and data analysts remained blinded to the types of treatment from the time of randomization until database lock. Randomization data were kept strictly confidential until the time of unblinding and were not accessible by anyone else involved in the study (except in the case of patient emergencies). The types of treatment were concealed by the use of study drugs that were all identical in packaging, labeling, schedule of administration, and appearance.

\section{Statistical Methods}

Patients who were administered at least one dose of the study drug, and had at least one safety evaluation after baseline, were considered for the safety analysis (the 'safety population'). The efficacy analysis was based on the full analysis set (FAS) defined as 'all randomized patients who received at least one dose of the study drug and had both baseline assessment and at least one post-baseline assessment for any of the efficacy variables'.

Statistical analyses were performed using SAS software (version 9.2). To meet the primary endpoint, the difference in the proportion of patients with AEs leading to discontinuation during the 24-week study period had to fall between the prespecified acceptance range $(-9.0,9.0 \%)$, and was evaluated in the safety population. If the observed difference fell within this range, the 1-step titration group was determined to be as tolerable as the 3-step titration group. The proportion of patients with AEs leading to discontinuation and its $95 \%$ confidence interval (CI) for both groups were calculated using the exact method, and the treatment difference in the proportion was also presented.

The study was not designed to show statistical significance for treatment group comparisons in any efficacy variables. Changes from baseline to weeks 8, 16 and 24 on the ADAS-J cog and changes from baseline to week 24 on the MMSE were assessed by analysis of covariance (ANCOVA), with treatment groups as a factor and baseline values as a covariate. Estimates [least squares (LS) mean] and 95\% CI of the mean for each treatment group as well as the treatment group differences were reported.

For the J-CGIC at weeks 4, 8, 12, 16, 20 and 24, the point estimates and 95\% CIs were provided by the treatment group, using the normal approximation to the binomial distribution, for the proportions of patients who were assessed to have no worsening (patients who were assessed either as 'markedly improved', 'improved', 'slightly improved' or 'no change') and improvement (patients who were assessed either as 'markedly improved', 'improved' or 'slightly improved'), respectively. Point estimates and 95\% CIs for the treatment group difference in the proportions of patients with no worsening and with improvement, respectively, were also reported for each visit, using the normal approximation to the binomial distribution. 


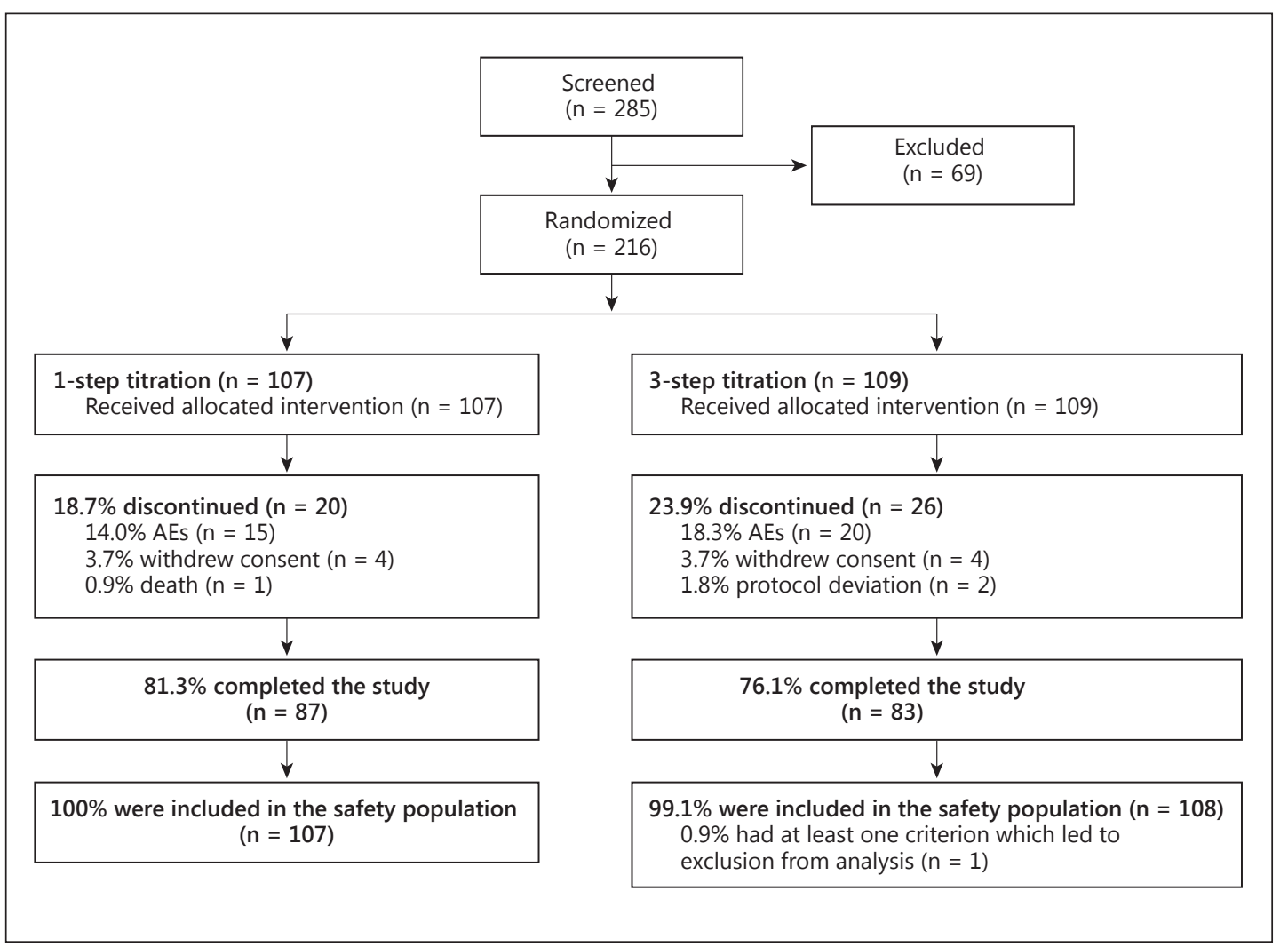

Fig. 1. Study design and patient flow through the study.

Missing values were handled via the last observation carried forward (LOCF) method. Analysis was also conducted using observed case (OC) to confirm the robustness of results. The missing values up to week 24 were imputed using the LOCF method when ADAS-J cog and J-CGIC total scores were missing. The missing values of the baseline observation were not imputed. The missing scale items were not imputed and were handled as missing values for ADAS-J cog and MMSE total scores if at least one scale item was missing.

AEs were standardized using the Medical Dictionary for Regulatory Activities terminology. They were indicated by preferred term unless otherwise specified.

The concomitant medications and non-drug therapies that were started on or after the first dose of the study drug were coded using the World Health Organization Drug Reference List that employs the Anatomical Therapeutic Chemical classification system.

\section{Results}

\section{Participants}

Of 216 patients who were randomized, 107 were allocated to the 1-step titration group and 109 to the 3 -step titration group. All randomized patients received the study drug. A total of 170 patients completed the study (1-step, $n=87 ; 3$-step, $n=83$ ). Slightly more patients in the 3-step titration group [23.9\% (26/109)] discontinued the study compared to the 1-step titration group [18.7\% (20/107)]. In both groups, AEs were the most frequent reason for discontinuation, followed by withdrawal of consent (fig. 1). All but one of the randomized 
Nakamura et al.: Different Titration Schemes of the Rivastigmine Patch in Japanese Patients with Mild to Moderate Alzheimer's Disease

Table 2. Baseline demographics and background characteristics (safety population)

\begin{tabular}{|c|c|c|c|}
\hline & $\begin{array}{l}\text { 1-step titration } \\
(\mathrm{n}=107)\end{array}$ & $\begin{array}{l}\text { 3-step titration } \\
(\mathrm{n}=108)\end{array}$ & $\begin{array}{l}\text { Total } \\
(n=215)\end{array}$ \\
\hline Female patients, $\%$ & 64.5 & 70.4 & 67.4 \\
\hline \multicolumn{4}{|l|}{ Age, years } \\
\hline Mean \pm SD & $77.5 \pm 6.54$ & $77.6 \pm 5.89$ & $77.5 \pm 6.21$ \\
\hline Range & $56-85$ & $56-85$ & $56-85$ \\
\hline \multicolumn{4}{|l|}{ Weight, kg } \\
\hline Mean \pm SD & $49.58 \pm 10.291$ & $49.24 \pm 9.367$ & $49.41 \pm 9.816$ \\
\hline Range & $30.9-77.9$ & $33.0-73.0$ & $30.9-77.9$ \\
\hline \multicolumn{4}{|c|}{ Time since physician first diagnosed AD symptoms, years } \\
\hline Mean \pm SD & $1.05 \pm 1.752$ & $1.11 \pm 1.893$ & $1.08 \pm 1.821$ \\
\hline Range & $0.0-9.5$ & $0.0-10.0$ & $0.0-10.0$ \\
\hline \multicolumn{4}{|l|}{ Living situation, $\%$} \\
\hline Living with caregiver/other individual & 97.2 & 97.2 & 97.2 \\
\hline Assisted living/group home & 0.0 & 0.9 & 0.5 \\
\hline Nursing home/long-term institution & 1.9 & 0.9 & 1.4 \\
\hline Other & 0.9 & 0.9 & 0.9 \\
\hline \multicolumn{4}{|l|}{ Formal education, years } \\
\hline Mean \pm SD & $10.5 \pm 2.64$ & $10.8 \pm 3.04$ & $10.6 \pm 2.85$ \\
\hline Range & $3-18$ & $6-21$ & $3-21$ \\
\hline \multicolumn{4}{|l|}{ Baseline MMSE score } \\
\hline Mean \pm SD & $17.2 \pm 2.51$ & $16.9 \pm 2.95$ & $17.1 \pm 2.74$ \\
\hline Range & $10-20$ & $10-20$ & $10-20$ \\
\hline \multicolumn{4}{|l|}{ Concurrent use of memantine, $\%$} \\
\hline Yes & 9.3 & 7.4 & 8.4 \\
\hline No & 90.7 & 92.6 & 91.6 \\
\hline
\end{tabular}

patients were included in the safety analysis (1-step, $n=107 ; 3$-step, $n=108$ ). Of these 215 patients, 209 comprised the FAS (1-step, $n=104$; 3-step, $n=105$ ) with 6 patients $(3$ in each group) excluded due to lack of post-baseline efficacy assessments.

Baseline demographics and background characteristics are summarized in table 2 . The majority of the safety population were female [67.4\% (145/215)]. The overall mean age \pm SD of the safety population was $77.5 \pm 6.21$ years and the weight was $49.4 \pm 9.82 \mathrm{~kg}$ (range, 30.9-77.9). The overall mean MMSE total score \pm SD at baseline was $17.1 \pm 2.74$ points. Of all patients, $8.4 \%(18 / 215)$ were using memantine at baseline. Treatment groups were well matched for both demographics and background characteristics.

\section{Dosing}

The mean duration of exposure to the study drug was comparable between treatment groups, and was approximately 21 weeks in both groups. The treatment retention rate was comparable in both groups [1-step, 71.0\% (76/107); 3-step, 69.4\% (75/108)].

\section{Concomitant Medications}

The use of concomitant medications active on or after the start of the study drug is presented in table 3 . The most frequently (at least $30 \%$ of patients, safety population) received concomitant medication was mucopolysaccharide polysulfuric acid ester in both treatment groups. 
Table 3. Number of patients with the most frequently (at least $10 \%$ in any group) received concomitant medications active on or after the start of the study drug (safety population)

\begin{tabular}{llc}
\hline Preferred term & $\begin{array}{l}\text { 1-step titration } \\
(\mathrm{n}=107)\end{array}$ & $\begin{array}{l}\text { 3-step titration } \\
\text { (n=108) }\end{array}$ \\
\hline Mucopolysaccharide polysulfuric acid ester & $37(34.6)$ & $39(36.1)$ \\
Amlodipine besilate & $16(15.0)$ & $25(23.1)$ \\
Loxoprofen sodium & $13(12.1)$ & $11(10.2)$ \\
Valisone-G & $13(12.1)$ & $14(13.0)$ \\
Rebamipide & $10(9.3)$ & $12(11.1)$ \\
Candesartan cilexetil & $7(6.5)$ & $11(10.2)$ \\
Magnesium oxide & $6(5.6)$ & $11(10.2)$ \\
\hline
\end{tabular}

Values are shown as $\mathrm{n}(\%)$.

Table 4. Proportion of patients having an AE leading to study drug discontinuation during the double-blind treatment period (safety population)

$\begin{array}{lll}\begin{array}{l}\text { 1-step titration } \\ (\mathrm{n}=107)\end{array} & \text { 3-step titration } & \text { 1-step titration - } \\ & (\mathrm{n}=108) & \text { 3-step titration }(\mathrm{n}=215)\end{array}$

Patients having an AE leading

to study drug discontinuation $16(15.0)[8.8,23.1] 20(18.5)[11.7,27.1] \quad-3.6[-17.0,9.6]$

Values are shown as n (\%) [95\% CI]. 95\% CIs are calculated using the exact method.

Tolerability and Safety Assessments

Proportion of Patients with AEs Leading to Discontinuation: Primary Endpoint

The proportion of patients with AEs leading to discontinuation during the double-blind treatment period was $15.0 \%(16 / 107)$ in the 1-step titration group and $18.5 \%(20 / 108)$ in the 3-step titration group. The observed between-treatment difference in the proportion of discontinuation due to AEs (1-step titration group - 3-step titration group) was -3.6\% (95\% CI: -17.0, 9.6). The point estimate of the observed difference fell between the prespecified acceptance range $(-9.0,9.0 \%)$, showing a similar tolerability between the two treatment groups, as defined in the study protocol (table 4).

Deaths, SAEs, Discontinuation due to AEs

Death was reported in 1 patient in the 1-step titration group. The specific cause of death remained unknown and was suspected by the investigator to be related to the study drug. Eight patients in the 1-step titration group and 10 patients in the 3-step titration group experienced SAEs (excluding death) (table 5). All SAEs by preferred term including death were isolated events which occurred in 1 patient each. Of nonfatal SAEs, those considered to be related to the study drug by the investigator were bradycardia in the 1-step titration group and abnormal liver function test, decreased appetite and dyslalia in the 3-step titration group. All of these 4 patients recovered as a result of medical treatment or study drug interruption, reduction or discontinuation. The most frequent AEs leading to discontinuation of the study drug were application site events (application site erythema and application site pruritus), with similar incidence in both treatment groups (table 6). 
Table 5. Number of patients who died, had SAEs, discontinued treatment due to AEs or SAEs or decreased/ interrupted the study drug due to AEs (safety population)

\begin{tabular}{lcc}
\hline Preferred term & 1-step titration $(\mathrm{n}=107)$ & 3-step titration $(\mathrm{n}=108)$ \\
\hline Death & $1(0.9)$ & $0(0.0)$ \\
SAEs $^{\mathrm{a}}$ & $9(8.4)$ & $10(9.3)$ \\
Discontinued due to AEs & $16(15.0)$ & $20(18.5)$ \\
Discontinued due to SAEs & $5(4.7)$ & $4(3.7)$ \\
Decreased/interrupted study drug due to AEs & $29(27.1)$ & $26(24.1)$ \\
\hline
\end{tabular}

The same patient may appear in more than one category. ${ }^{\text {a }}$ Includes death.

Table 6. Number of patients with AEs leading to discontinuation of the study drug (safety population)

\begin{tabular}{lcc}
\hline Preferred term & $\begin{array}{l}1 \text {-step titration } \\
(\mathrm{n}=107)\end{array}$ & $\begin{array}{l}\text { 3-step titration } \\
(\mathrm{n}=108)\end{array}$ \\
\hline Total number of patients with an AE & $16(15.0)$ & $20(18.5)$ \\
Bradycardia & $1(0.9)$ & $0(0.0)$ \\
Atrial fibrillation & $0(0.0)$ & $1(0.9)$ \\
Gastritis & $1(0.9)$ & $0(0.0)$ \\
Nausea & $1(0.9)$ & $1(0.9)$ \\
Application site erythema & $5(4.7)$ & $5(4.6)$ \\
Application site pruritus & $4(3.7)$ & $3(2.8)$ \\
Application site dermatitis & $1(0.9)$ & $1(0.9)$ \\
\hline Death & $1(0.9)$ & $0(0.0)$ \\
Spinal compression fracture & $1(0.9)$ & $0(0.0)$ \\
Cervical vertebral fracture & $0(0.0)$ & $1(0.9)$ \\
Pelvic fracture & $0(0.0)$ & $1(0.9)$ \\
Rib fracture & $0(0.0)$ & $1(0.9)$ \\
ECG QT prolonged & $0(0.0)$ & $2(1.9)$ \\
ECG T-wave inversion & $0(0.0)$ & $1(0.9)$ \\
\hline Decreased appetite & $1(0.9)$ & $0(0.0)$ \\
Breast cancer & $1(0.9)$ & $0(0.0)$ \\
Bladder cancer & $0(0.0)$ & $1(0.9)$ \\
Malignant lung neoplasm & $0(0.0)$ & $1(0.9)$ \\
Cerebral hemorrhage & $1(0.9)$ & $0(0.0)$ \\
Dizziness & $1(0.9)$ & $0(0.0)$ \\
Alzheimer's type dementia & $0(0.0)$ & $1(0.9)$ \\
\hline Dyslalia & $0(0.0)$ & $1(0.9)$ \\
Sleep phase rhythm disturbance & $0(0.0)$ & $1(0.9)$ \\
Irritability & $1(0.9)$ & $0(0.0)$ \\
Persecutory delusion & $0(0.0)$ & $1(0.9)$ \\
Restlessness & $0(0.0)$ & $1(0.9)$ \\
Dermatitis contact & $1(0.9)$ & $2(1.9)$ \\
Eczema & $0(0.0)$ & $1(0.9)$ \\
\hline Values are shown as n (\%). & & \\
\hline & & \\
& & \\
\hline
\end{tabular}


Table 7. Number of patients with most frequent AEs (at least $2 \%$ in any group) regardless of study drug relationship (safety population)

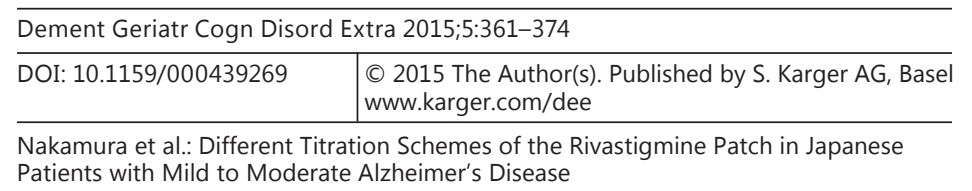

\begin{tabular}{lll}
\hline Preferred term & $\begin{array}{l}\text { 1-step titration } \\
(\mathrm{n}=107)\end{array}$ & $\begin{array}{l}\text { 3-step titration } \\
(\mathrm{n}=108)\end{array}$ \\
\hline Total number of patients with an AE & $85(79.4)$ & $85(78.7)$ \\
Application site pruritus & $24(22.4)$ & $24(22.2)$ \\
Application site erythema & $17(15.9)$ & $17(15.7)$ \\
Dermatitis contact & $13(12.1)$ & $12(11.1)$ \\
Nasopharyngitis & $9(8.4)$ & $12(11.1)$ \\
Decreased appetite & $5(4.7)$ & $6(5.6)$ \\
\hline Diarrhea & $5(4.7)$ & $3(2.8)$ \\
Vomiting & $5(4.7)$ & $3(2.8)$ \\
Application site dermatitis & $4(3.7)$ & $3(2.8)$ \\
Nausea & $4(3.7)$ & $6(5.6)$ \\
Restlessness & $4(3.7)$ & $1(0.9)$ \\
\hline Decreased weight & $4(3.7)$ & $2(1.9)$ \\
Application site rash & $3(2.8)$ & $6(5.6)$ \\
Constipation & $3(2.8)$ & $2(1.9)$ \\
Hypertension & $3(2.8)$ & $5(4.6)$ \\
Influenza & $3(2.8)$ & $0(0.0)$ \\
\hline Contusion & $2(1.9)$ & $3(2.8)$ \\
Eczema & $2(1.9)$ & $3(2.8)$ \\
Gastroenteritis & $2(1.9)$ & $4(3.7)$ \\
Back pain & $1(0.9)$ & $3(2.8)$ \\
ECG QT prolonged & $1(0.9)$ & $3(2.8)$ \\
\hline Values are shown as n (\%). & & \\
\hline
\end{tabular}

Incidence of AEs

During the study, $79.4 \%$ of patients in the 1 -step titration group and $78.7 \%$ of patients in the 3-step titration group had at least one AE (table 7). The most common AEs in both groups were application site events (application site pruritus, application site erythema and dermatitis contact), and most events were mild and moderate in severity. The overall incidence of gastrointestinal events (diarrhea, vomiting and nausea) was similar between the treatment groups [1-step, 11.2\% (12/107); 3-step, 9.3\% (10/108)]. The reported AEs and the incidences (including gastrointestinal events) during the entire study period were similar between the treatment groups.

For the titration period (weeks 1-16), the overall incidence of AEs reported was similar in both treatment groups [1-step, 68.2\% (73/107); 3-step, 67.6\% (73/108)]. This result shows that most of the AEs reported during the entire study period were first reported in the titration period. The most commonly reported AEs during the titration period were the same as those reported during the entire study period (online suppl. table 1; for all online suppl. material, see www.karger.com/doi/10.1159/000439269).

During the first 4 weeks (weeks 1-4), the reported AEs and the incidences were similar between the treatment groups [1-step, 24.3\% (26/107)]; 3-step, 27.8\% (30/108)], although the starting dose of rivastigmine was different (1-step, $9 \mathrm{mg} /$ day; 3-step, $4.5 \mathrm{mg} /$ day).

There were no major differences between treatments in change from baseline for pulse rate, systolic or diastolic blood pressure, or ECG recordings. The frequency of clinically notable changes in weight $(\geq 7 \%$ decrease from baseline) was similar between the treatment groups [1-step, 10.3\% (11/107); 3-step, 12.0\% (13/108)]. Patients who decreased in weight (reported as an AE) did not experience gastrointestinal events such as nausea, vomiting and diarrhea. 
Nakamura et al.: Different Titration Schemes of the Rivastigmine Patch in Japanese Patients with Mild to Moderate Alzheimer's Disease

Fig. 2. LS mean (and 95\% CI) changes from baseline on the ADAS-J cog (FAS-LOCF). LS mean and $95 \% \mathrm{CI}$ are based on the ANCOVA model including titration group as a factor and baseline ADAS-J cog score as a covariate.

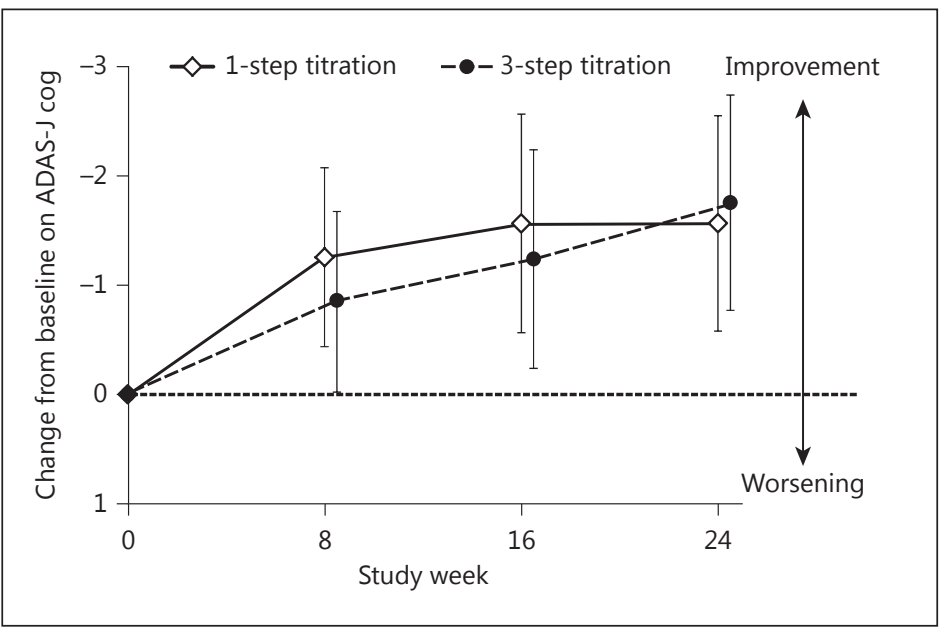

\section{Efficacy Assessments: Secondary Endpoints}

ADAS-J cog

The LS mean change from baseline on the ADAS-J cog of the FAS-LOCF showed a decrease in both treatment groups at every time point, indicating a cognitive improvement (fig. 2). The upper bound of $95 \% \mathrm{CI}$ for the LS mean was lower than 0 at each time point in each treatment group. The point estimate of the LS mean difference (1-step titration group - 3-step titration group) indicated a numerical advantage of the 1-step treatment group at week 8 [ -0.4 points (95\% CI: $-1.6,0.8)]$. Week 8 was the first time point when the effective dose of $18 \mathrm{mg} /$ day was administered in the 1-step treatment group, whereas $9 \mathrm{mg} /$ day was administered in the 3 -step treatment group. The mean change \pm SD from baseline to week 24 on the ADAS-J cog was $-1.6 \pm 4.66$ points in the 1 -step titration group and $-1.8 \pm 5.58$ points in the 3 -step titration group. The treatment difference of LS mean was 0.2 points (95\% CI: $-1.2,1.6)$ at week 24 , indicating a numerical advantage in favor of the 3-step treatment group (online suppl. table 2). The results from the FAS-OC were consistent with those obtained from the FAS-LOCF.

\section{MMSE}

The mean change \pm SD from baseline at week 24 on the MMSE of the FAS-LOCF was 0.6 \pm 2.91 points in the 1 -step titration group and $0.5 \pm 3.15$ points in the 3 -step titration group, showing a slight cognitive improvement in both groups. The treatment difference of LS mean was 0.1 points $(95 \% \mathrm{CI}:-0.8,1.0)$ at week 24 , indicating a similar performance between the two treatment groups (online suppl. table 3).

\section{J-CGIC}

Figure 3 shows the changes in the percentage (and 95\% CI) of patients with no worsening (a total of 'markedly improved', 'improved', 'slightly improved' and 'no change') and with improvement (a total of 'markedly improved', 'improved' and 'slightly improved') over the 24 -week study period for the FAS-LOCF. The 95\% CIs of the individual treatment groups overlapped at every time point, indicating a similar performance between the two treatment groups. However, the numerical advantage for the 1-step titration group compared to the 3 -step titration group was most prominent in the percentage of patients with improvement at week 8 [8.9\% (95\% CI: $-4.3,22.1)]$. The percentage of patients with no worsening and the percentage of those with improvement at week 24 indicated a numerical advantage of the 1-step titration group over the 3 -step titration group (online suppl. tables 4, 5). Similar results as for the J-CGIC were observed for the FAS-OC. 
Fig. 3. Percentages of patients (and 95\% CI) with no worsening and with improvement in the JCGIC (FAS-LOCF). 95\% CI is based on the normal approximation.
Nakamura et al.: Different Titration Schemes of the Rivastigmine Patch in Japanese Patients with Mild to Moderate Alzheimer's Disease

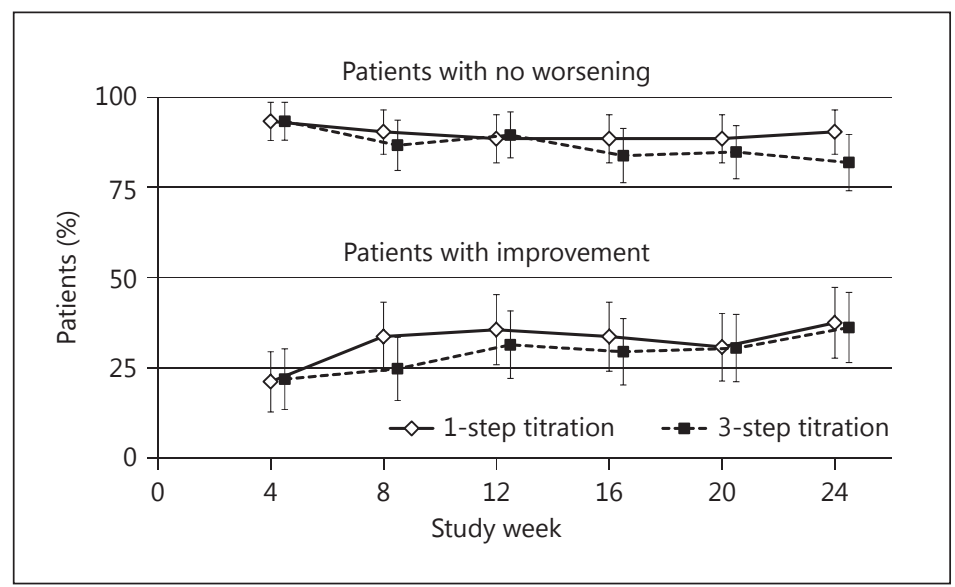

\section{Discussion}

Treatment with the 1-step titration scheme of the rivastigmine patch for 24 weeks provided similar tolerability to the 3-step titration, a currently available titration method of the drug for AD in Japan, in participants of mild to moderate AD on the basis of a score of $\geq 10$ and $\leq 20$ on the MMSE.

The study found that the difference between the two groups in treatment discontinuation rates due to AEs during the 24-week treatment period was small and fell within the predefined acceptance range of $9 \%$. As with previous clinical studies with the rivastigmine patch or any medical patch in Japan, skin application site reactions were the major cause of treatment discontinuation for both treatment groups [2]. These typically take the form of mild erythema and pruritus and do not present a serious medical problem [9]. In this study, the incidence of severe skin application site reactions was low $(<3 \%$ of patients in both treatment groups).

Gastrointestinal adverse effects, such as nausea, vomiting and diarrhea, are common to all AChE inhibitors, particularly during the titration phase [10-12]. In this study, the overall incidence of gastrointestinal AEs (nausea, vomiting and diarrhea) in both treatment groups was low and was comparable between the two groups (about $10 \%$ for both groups). A similar percentage of patients who met the prespecified clinically notable weight loss criteria at any time during the study ( $\geq 7 \%$ decrease from baseline) was seen between the groups (about $10-12 \%$ in each group). A small and similar number of patients reported weight loss as an $\mathrm{AE}$ in both groups [1-step, 3.7\% (4/107); 3-step, 1.9\% (2/108)]. About 17\% of the total number of patients discontinued treatment during the 24-week study period. The treatment retention rate during the last 8 weeks was confirmed to be similar for both groups (1-step, $71.0 \%$ vs. 3 -step, 69.4\%), demonstrating sufficient exposure to rivastigmine regardless of the titration method chosen.

The overall safety results in the current study, including those at weeks 4 (initial dose period), 16 (end of the titration period) and 24 (end of the 8-week maintenance period), found the 1-step titration method to demonstrate comparable tolerability with the 3-step titration method. Particularly, the safety profile of the 1-step titration method in the first 4-week period, where a starting dose of $9 \mathrm{mg} /$ day of the rivastigmine patch was administered, was similar to that in the 3-step titration method, starting with $4.5 \mathrm{mg} /$ day for that period. These results could be interpreted that 1-step titration can provide patients additional benefits of reaching the effective dose faster, while maintaining a comparable safety 
Nakamura et al.: Different Titration Schemes of the Rivastigmine Patch in Japanese Patients with Mild to Moderate Alzheimer's Disease

profile with the 3-step titration method. In other words, the 1-step titration method could fulfill a practical clinical need in patients treated with the rivastigmine patch compared to the currently available 3-step titration, which needs a fairly lengthy titration period of up to at least 12 weeks before reaching the effective dose. This may address concerns from physicians and/or caregivers that clinical signs and symptoms of the patients may not be sufficiently treated during a long titration period. Therefore, the addition of the 1-step titration method to the approved dosing regimen is clinically relevant as it can provide patients a greater chance of receiving treatment with a therapeutic dose earlier.

This study was conducted in a clinically relevant population of patients with mild to moderate AD with a score of $\geq 10$ and $\leq 20$ on the MMSE. As the study was carried out for patients of both sexes, aged over 50 years and at different levels of prior treatment (including patients who had never been treated with AD drugs, those treated with memantine monotherapy and those with a 4-week washout period of AChE inhibitors), the results indicate that a relatively wide range of patients with $A D$ would benefit from the new titration method.

The study found that the two titration methods are very similar in efficacy. The 1-step titration method of rivastigmine led to a similar improvement in ADAS-J cog and to a similar percentage of 'improvement' in J-CGIC, reaching similar levels to those in the 3-step titration for all evaluated time points except for week 8 on these two measurements, when numerical advantages for the 1-step over the 3-step titration method were observed. The reason could be that patients in the 1-step titration group at week 8 had already received the effective dose of rivastigmine (18 $\mathrm{mg} /$ day), whereas the 3 -step titration group were still receiving the titration dose of the study drug ( $9 \mathrm{mg} /$ day). The results of J-CGIC in the course of treatment might have better clinical effects in functional and behavioral areas.

This study has certain limitations. Firstly, efficacy outcomes (ADAS-J cog, MMSE, J-CGIC) were secondary endpoints, and the study was not designed to show statistically significant between-treatment differences at any time point for these parameters. Another limitation of this study includes the relatively short treatment period to evaluate long-term efficacy of the 1-step titration method in comparison with the 3-step titration as medical treatments for AD are usually extended over longer periods of time. We set the duration of 24-week treatment to evaluate the tolerability of the new titration method, the primary objective of the study.

The 1-step titration method of the rivastigmine patch was as well tolerated as the 3-step titration method. Adding the 1-step titration method to the existing standard regimen of the rivastigmine patch is clinically relevant as it enables physicians to choose either regimen depending on patients' signs and symptoms and/or their tolerability of the drug.

In conclusion, the present study showed that the 1-step titration method of the rivastigmine patch might be a useful $\mathrm{AD}$ treatment as it can provide an additional option with the benefit of faster titration to the effective dose than the 3-step titration.

\section{Acknowledgements}

The AD study for which the current data were collected and the current data analyses were sponsored by Novartis and Ono Pharmaceutical Co., Ltd. We thank Makoto Shimozawa who belonged to Novartis Pharma K.K. as a clinical scientist, and we also thank the CNS Medical Franchise Department as well as the Medical and Scientific Communication and Document Department, Novartis K.K. and Ono Pharmaceutical Co., Ltd., for providing medical editorial assistance with this paper. 


\section{Disclosure Statement}

Yu Nakamura acted as the external medical expert in this study. In the last 3 years, he has received fees for speaking, consultancy, manuscript writing or document supervision, and his department has received scholarship grants from Novartis Pharma K.K., Ono Pharmaceutical Co., Ltd., Daiichi Sankyo, Takeda, Janssen, Eisai, Pfizer, Mochida, Mitsubishi Tanabe, Yoshitomi, Kowa, Toyama Chemical, Meiji Seika, Astellas, Dainippon Sumitomo, Boehringer Ingelheim, GlaxoSmithKline, MSD, AstraZeneca, Biogenidec Japan, Shionogi, Eli Lilly Japan, Otsuka, FUJIFILM RI and AbbVie GK. Akira Homma was the coordinating investigator for this study. In the last 3 years, he has received fees for speaking, consultancy, manuscript writing or document supervision from Novartis Pharma K.K., Ono Pharmaceutical Co., Ltd., Daiichi Sankyo, Takeda, Janssen, Eisai, Mitsubishi Tanabe, Kowa, Toyama Chemical, Astellas, Dainippon Sumitomo, Boehringer Ingelheim, GlaxoSmithKline, MSD, Shionogi and Otsuka. Both of these authors have received no payment for the preparation of the manuscript. Christine Strohmaier is a full-time employee of Novartis Pharma AG. Kaoru Tamura and Naoko Kataoka are full-time employees of Novartis Pharma K.K. Masayuki Nakano, Shoichiro Oda and Kazuma Nishimura are full-time employees of Ono Pharmaceutical Co., Ltd.

\section{References}

1 Kurz A, Farlow M, Lefèvre G: Pharmacokinetics of a novel transdermal rivastigmine patch for the treatment of Alzheimer's disease: a review. Int J Clin Pract 2009;63:799-805.

-2 Nakamura Y, Imai Y, Shigeta M, Graf A, Shirahase T, Kim H, Fujii A, Mori J, Homma A: A 24-week, randomized, double-blind, placebo-controlled study to evaluate the efficacy, safety and tolerability of the rivastigmine patch in Japanese patients with Alzheimer's disease. Dement Geriatr Cogn Disord Extra 2011;1:163-179.

-3 McKhann G, Drachman D, Folstein M, Katzman R, Price D, Stadlan EM: Clinical diagnosis of Alzheimer's disease: report of the NINCDS-ADRDA Work Group under the auspices of Department of Health and Human Services Task Force on Alzheimer's Disease. Neurology 1984;34:939-944.

-4 Folstein MF, Folstein SE, McHugh PR: 'Mini-mental state'. A practical method for grading the cognitive state of patients for the clinician. J Psychiatr Res 1975;12:189-198.

5 Homma A, Fukuzawa K, Tsukada Y, Ishii T, Hasegawa K, Mohs RC: Development of a Japanese version of Alzheimer's disease assessment scale (ADAS) (in Japanese). Jpn J Geriatr Psychiatry 1992;3:647-655.

6 Homma A, Takeda M, Imai Y, Udaka F, Hasegawa K, Kameyama M, Nishimura T; E2020 Study Group: Clinical efficacy and safety of donepezil on cognitive and global function in patients with Alzheimer's disease. A 24-week, multicenter, double-blind, placebo-controlled study in Japan. Dement Geriatr Cogn Disord 2000;11: 299-313.

7 Winblad B, Cummings J, Andreasen N, Grossberg G, Onofrj M, Sadowsky C, Zechner S, Nagel J, Lane R: A sixmonth double-blind, randomized, placebo-controlled study of a transdermal patch in Alzheimer's disease rivastigmine patch versus capsule. Int J Geriatr Psychiatry 2007;22:456-467.

-8 Sadowsky CH, Dengiz A, Meng X, Olin JT; US38 Study Group: Switching from oral donepezil to rivastigmine transdermal patch in Alzheimer's disease: 20-week extension phase results. Prim Care Companion J Clin Psychiatry 2010;12:pii:PCC.09m00852oli.

-9 Darreh-Shori T, Jelic V: Safety and tolerability of transdermal and oral rivastigmine in Alzheimer's disease and Parkinson's disease dementia. Expert Opin Drug Saf 2010;9:167-176.

10 Bassil N, Grossberg GT: Novel regimens and delivery systems in the pharmacological treatment of Alzheimer's disease. CNS Drugs 2009;23:293-307.

11 Gauthier S: Cholinergic adverse effects of cholinesterase inhibitors in Alzheimer's disease: epidemiology and management. Drug Aging 2001;18:853-862.

12 Birks J, Evans JG, Iakovidou V, Tsolaki M, Holt FE: Rivastigmine for Alzheimer's disease (review). Cochrane Database Syst Rev 2009;2:CD001191. 